\title{
Unit of Energy Density
}

National Cancer Institute

\section{Source}

National Cancer Institute. Unit of Energy Density. NCI Thesaurus. Code C70442.

A unit used to express the amount of energy stored in a given system or region of space per unit volume. 